\title{
Gain-of-function PDGFRA mutations, earlier reported in gastrointestinal stromal tumors, are common in small intestinal inflammatory fibroid polyps. A study of 60 cases
}

\author{
Jerzy Lasota ${ }^{1}$, Zeng-Feng Wang ${ }^{2}$, Leslie H Sobin ${ }^{3}$ and Markku Miettinen ${ }^{1}$ \\ ${ }^{1}$ Department of Soft Tissue Pathology, Armed Forces Institute of Pathology, Washington, DC, USA; \\ ${ }^{2}$ Department of Scientific Laboratories, Armed Forces Institute of Pathology, Washington, DC, USA and \\ ${ }^{3}$ Division of Gastrointestinal Pathology, Armed Forces Institute of Pathology, Washington, DC, USA
}

\begin{abstract}
The inflammatory fibroid polyp is a rare benign lesion occurring throughout the digestive tract. It usually forms a solitary mass, characterized by a proliferation of fibrovascular tissue infiltrated by a variable number of inflammatory cells. The etiology of this lesion is unknown and conflicting histogenetic theories have been proposed. Recently, mutations in platelet-derived growth factor receptor (PDGFRA) and PDGFRA expression were reported in gastric inflammatory fibroid polyps. In this study, PDGFRA exons 12, 14, and 18 were screened for activating mutations in 60 small intestinal inflammatory fibroid polyps. In addition, the PDGFRA expression was evaluated immunohistochemically. Mutations in PDGFRA were identified in 33 of 60 (55\%) cases, whereas 95\% expressed PDGFRA. There were 26 deletions, three deletion-insertions, duplication, and single nucleotide substitution in exon 12, and a single nucleotide substitution and deletion in exon 18. The majority ( $n=23)$ of exon 12 deletions were 1837_1851del leading to S566_E571delinsR. However, 1835_1852delinsCGC leading to the same S566_E571delinsR, were found in two tumors. Three inflammatory fibroid polyps had 1836_1850del leading to S566_E571delinsK. A complex deletion-insertion affecting a similar region (1837_1856delinsGATTGATGATC) and leading to S566_I573delinsRIDDL was identified once. In addition, duplication and single nucleotide substitution were found $5^{\prime}$ to the common inflammatory fibroid polyp mutational 'hot spot'. These mutations consist of 1808_1828dup leading to I557_E563dup, and 1821T $>$ A resulting in $561 \mathrm{~V}>\mathrm{D}$ substitution. A 2664A $>$ T and 2663_2674del leading to 842D $>V$ and D842_H845del, respectively, were identified in exon 18. Similar gain-of-function PDGFRA mutations reported in gastrointestinal stromal tumors have been considered to be a driving pathogenetic force. This study showed consistent expression and common mutational activation of PDGFRA in small intestinal inflammatory fibroid polyps as in their gastric counterparts, and these lesions should be considered PDGFRA-driven benign neoplasms. We also suggest that these polyps may develop from earlier described PDGFRA-positive mesenchymal cells distributed along the villus membrane after oncogenic PDGFRA activation.

Modern Pathology (2009) 22, 1049-1056; doi:10.1038/modpathol.2009.62; published online 15 May 2009
\end{abstract}

Keywords: inflammatory fibroid polyp; PDGFRA; PDGFRA-positive villus clusters; mutation; immunohistochemistry

Correspondence: Dr J Lasota, MD, Department of Soft Tissue and Orthopedic Pathology, Armed Forces Institute of Pathology (Building 54), 6825 16th Street, N.W., Washington, DC 203066000, USA.

E-mail: lasota@afip.osd.mil

The opinions and assertions contained herein are the expressed views of the authors and are not to be construed as official or reflecting the views of the Departments of the Army or Defense. This study was partially supported by the American Registry of Pathology.

Received 26 November 2008; revised 24 February 2009; accepted 25 February 2009; published online 15 May 2009
The inflammatory fibroid polyp is a solitary, intraluminal polypoid lesion of the gastrointestinal tract, characterized by a proliferation of highly vascular fibrous tissue and infiltrated by a variable number of inflammatory cells. Inflammatory fibroid polyp was first reported by Vanek in 1949, as 'submucosal granuloma with eosinophilic infiltration'. ${ }^{1}$ Subsequent reports introduced other names, such as eosinophilic granuloma, gastric fibroma with eosinophilic infiltration, granuloma with eosinophils, 'hemangiopericytoma', inflammatory fibroid tumor, and inflammatory pseudotumor. ${ }^{2}$ 
However, only the term inflammatory fibroid polyp, coined by Helwig and Ranier ${ }^{3}$, has gained wide acceptance.

A variety of names used in the literature reflect different hypotheses on the etiology and histogenesis of inflammatory fibroid polyps. Exuberant host response to an unknown local injury, infection, and allergic reaction have been considered as possible etiological factor. ${ }^{4}$ However, the hamartomatous or neoplastic nature of the inflammatory fibroid polyp was suggested in the context of familial occurrence. ${ }^{5}$ Descriptive names such as 'self-limiting proliferation of histiocytes' were occasionally used without evidence of the true nature of these polyps. ${ }^{6}$ Despite many ultrastructural and immunohistochemical studies suggesting dendritic, fibroblastic, fibrohistiocytic, histiocytic, myofibroblastic, neural, and vascular differentiation, an origin of spindle cells comprising these polyps has remained enigmatic. ${ }^{7-12}$

A recent study of 23 gastric inflammatory fibroid polyps showed an expression of plateletderived growth factor receptor (PDGFRA) and oncogenic PDGFRA mutations in a majority of analyzed tumors, and suggested that the inflammatory fibroid polyp is a neoplasm driven by activated PDGFRA. ${ }^{13}$

The PDGFRA gene maps to chromosome 4q12 and encodes a transmembrane glycoprotein of type III receptor tyrosine kinase. This protein is highly homologous to KIT, and both genes might have evolved from the duplication of a common ancestral gene. Normally PDGFRA kinase is activated by its ligands, platelet-derived growth factors, but mutations can lead to ligand-independent kinase activation. ${ }^{14-17}$ Gain-of-function PDGFRA mutations have been reported in a subset of gastrointestinal stromal tumors (GISTs) of the stomach, often characterized by epithelioid cell morphology. ${ }^{18,19}$

In this study, the mutational status of PDGFRA and PDGFRA protein expressions was evaluated in 60 well-characterized small intestinal inflammatory fibroid polyps to gain an understanding of the similarities and differences between gastric and small intestinal examples of this entity.

\section{Materials and methods}

Formalin-fixed and paraffin-embedded inflammatory fibroid polyps of the small intestine were retrieved from the files of the Armed Forces Institute of Pathology (AFIP), Washington, DC, USA. Demographic, clinical and follow-up data were obtained according to the Institutional Review Board approval.

\section{Immunohistochemical Studies}

Expressions of KIT (CD117), CD34, smooth muscle actin, and desmin were evaluated immunohisto- chemically as reported earlier. ${ }^{20}$ The DOG-1 expression was tested using mouse monoclonal antibody (Clone K9, Leica Microsystem Inc., Bannockburn, IL, USA). The antibody dilution was 1:100. Immunohistochemical studies on PDGFRA expression were carried out with two antibodies, a rabbit polyclonal antibody, SC338 (Santa Cruz, Biotechnology Inc., Santa Cruz, CA, USA), and a monoclonal mouse antibody, MAB322 (R\&D Systems Inc., Minneapolis, MN, USA). The antibody dilutions were 1:400 and 1:50, respectively. Immunohistochemistry was carried out on a Ventana Benchmark autostainer (Ventana Medical Systems Inc., Tucson, AZ, USA) after heat-induced epitope retrieval.

\section{Molecular Genetic Studies}

Tumor DNA samples obtained from formalin-fixed paraffin-embedded tissues were screened for mutations in PDGFRA exons 12, 14, and 18 by PCR amplification and direct sequencing of PCR products, as reported earlier. ${ }^{19,21}$ Nomenclature of the mutations was based on the recommendations of the Human Genome Variation Society (http:// www.hgvs.org). Mutations at the protein level were deduced under the assumption that all changes identified at the genomic level involved one allele. The PDGFRA (ACO98587) sequence obtained from the National Center for Biotechnology Information (NCBI) at http://www.ncbi.nlm.nih.gov was used as a reference.

The initial screening for PDGFRA mutations showed 29 inflammatory fibroid polyps with PDGFR exon 12, 14, and 18 wild-type sequences. In these tumors, a substantial number of inflammatory and other non-neoplastic cells were identified in standard H\&E and in immunohistochemical evaluation as well (Figure 1a and b). As the presence of DNA template from normal cells can relatively decrease the proportion of mutated allele in the tumor, we used the following strategy to reduce the copy number of PDGFRA exon 12 and exon 18 wild-type sequences. Restriction maps of restriction endonuclease cleavage sites within PDGFRA exon 12 and exon 18 PCR amplification products were generated. Unique MspI, NsiI, and HinfI cleavage sites were identified inside the codons 567, 845, and 846, which were among the deleted codons in inflammatory fibroid polyps. Genomic DNA samples from tumors initially identified as PDGFRA wild type were digested with MspI (Fermentas International Inc., Burlington, Ontario, Canada), NsiI (New England Biolabs Inc., Ipswich, MA, USA), and HinfI (Fermentas) enzymes to reduce the copy number of PDGFRA exon 12 or exon 18 wild-type sequences. Restriction enzyme-cleaved DNA was extracted and evaluated again for PDGFRA exons 12 and 18 deletion/deletion-insertions by PCR amplification and direct sequencing. 


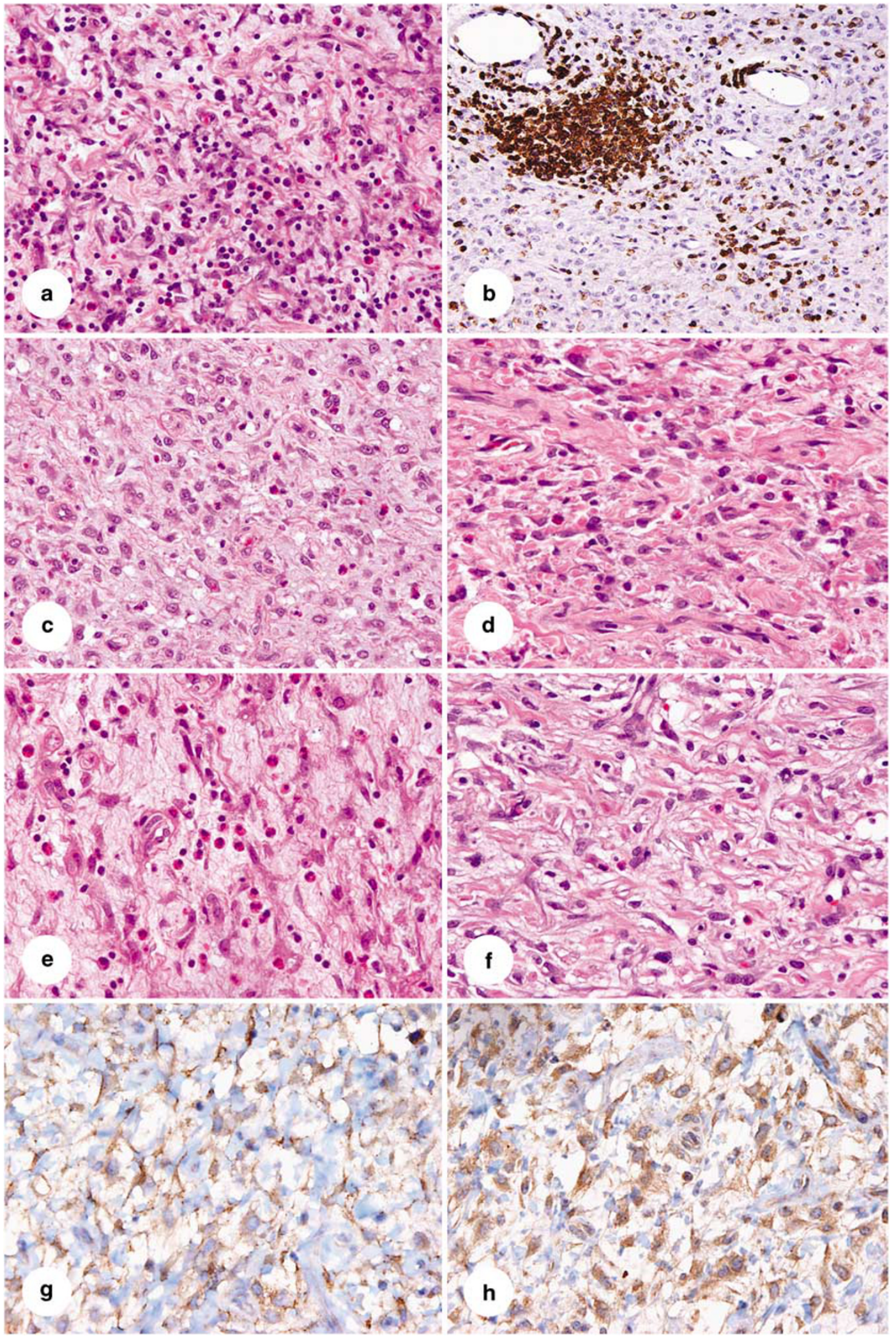

Figure 1 Inflammatory fibroid polyps can contain large numbers of inflammatory cells, especially eosinophils (a). These elements are highlighted as CD45 positive (b). (c-f) Histologically, inflammatory fibroid polyp is composed of polygonal to spindled cells in a highly vascular background. Collagenous (d) or edematous (e) matrix with capillary vessels (f) variably infiltrated by eosinophilic granulocytes and other inflammatory cells (c-f). Cytoplasmic expression of PDGFRA is common; immunohistochemistry with the polyclonal antibody, SC338 (g), and the monoclonal antibody, MAB322 (h). 


\section{Results}

\section{Clinicopathological Features}

The patient age varied from 13 to 83 years, with a median age of 54 years and a 1:1 male to female ratio. Data on the involved small intestinal segment were available in 32 cases. A total of 27 tumors (84\%) were located in the ileum, whereas five (16\%) were from the jejunum. The tumor size varied from 2 to $13 \mathrm{~cm}$ (median, $4.4 \mathrm{~cm}$ ), and $76 \%$ of the tumors measured 2-5 cm.

Histologically, the inflammatory fibroid polyps consisted of an admixture of dendritic-shaped or epithelioid to polygonal mesenchymal cells and inflammatory cells, especially eosinophilic granulocytes. Mitotic activity was scant and atypia was absent. There was a well-developed capillary network and a variably collagenous or myxoid extracellular matrix. Surface ulceration and underlying reactive myofibroblastic proliferation were common features. Representative histological images are shown in Figure 1c-f.

Complete or partial follow-up data were available in 16 cases. Seven patients were alive, with either no evidence of the disease $(n=5)$ or with tumor status unknown $(n=2)$ after 135-345 months (average follow-up: 223 months). Nine patients died of unrelated or unknown causes after 36-205 months (average follow-up: 142 months).

\section{Immunohistochemistry}

PDGFRA expression was evaluated using two antibodies, SC338 and MAB322. A total of 54 (90\%) of the cases showed strong, diffuse $(n=51)$, or focal $(n=3)$ PDGFRA positivity in both reactions. In three cases, PDGFRA expression was seen with one of two immunohistochemical stains. The remaining three tumors were negative with both antibodies. Thus, 95\% of the inflammatory fibroid polyps immunohistochemically expressed PDGFRA when combining results from both antibodies. CD34 was expressed diffusely in 11 inflammatory fibroid polyps and focally in three tumors in approximately 5 , 20, and $35 \%$ of tumor cells. Two polyps showed diffuse $(100 \%)$ or focal $(25 \%)$ smooth-muscle actin expression. All analyzed cases were negative for KIT, DOG-1, and desmin. Representative examples of immunostains are shown in Figure $1 \mathrm{~g}$ and $\mathrm{h}$.

\section{PDGFRA Mutations}

Mutations in PDGFRA were identified in 33 of 60 $(55 \%)$ analyzed inflammatory fibroid polyps. In four of these cases, PDGFRA exon 12 mutations were identified after the copy number of PDGFRA wildtype sequences was reduced by cleavage of normal allele with $M s p I$ restriction endonuclease.
There were 26 deletions, three deletion-insertions, one duplication, and one nucleotide substitution in exon 12. In exon 18, a single nucleotide substitution and deletion were identified. A majority $(n=23)$ of exon 12 deletions were 1837_1851del leading to S566_E571delinsR at the protein level. However, 1835_1852delinsCGC leading to the same S566_E571delinsR were found in two tumors. Three polyps had 1836_1850del leading to S566_E571delinsK. In one case, a complex deletion-insertion affecting a similar region (1837_1856delinsGATTGATGATC) and leading to S566_I573delinsRIDDL was identified. Two exon 12 mutations, a duplication and a single nucleotide substitution, were found $5^{\prime}$ to the common mutational 'hot spot' in inflammatory fibroid polyps. These mutations consist of 1808_1828dup leading to I557_E563dup, and $1821 \mathrm{~T}>\overline{\mathrm{A}}$ resulting in $561 \mathrm{~V}>\mathrm{D}$ substitution. Figure 2 shows a spectrum of PDGFRA exon 12 mutants. Exon 18 mutations included 2664A $>\mathrm{T}$ and 2663_2674del, leading to $842 \mathrm{D}>\mathrm{V}$ substitution and D842_H845del at the protein level, respectively.

\section{Discussion}

Inflammatory fibroid polyp is a rare benign lesion occurring throughout the digestive tract. ${ }^{1}$ Etiology and histogenesis of this lesion have remained enigmatic since a first series was reported by Vanek in 1949. However, a recent study of 23 gastric inflammatory fibroid polyps identified PDGFRA expression and PDGFRA-activating mutations, earlier seen only in a subset of gastric GISTs. This discovery has provided strong evidence of clonal proliferation and suggests a neoplastic nature of the inflammatory fibroid polyp. ${ }^{13}$

In this study, PDGFRA expression was detected immunohistochemically in $95 \%$ of small intestinal inflammatory fibroid polyps using two antibodies, a rabbit polyclonal and a mouse monoclonal. A great majority of inflammatory fibroid polyps showed similar results with both antibodies. None of the PDGFRA-negative tumors had PDGFRA mutations, although all of them displayed typical histological features. It is possible that in some cases, the level of PDGFRA expression is undetectable, but it is also possible that some small intestinal inflammatory fibroid polyps are driven by molecular mechanisms other than PDGFRA activation. Additional studies are necessary to address these hypotheses.

Although PDGFRA expression was detected in a great majority of small intestinal inflammatory fibroid polyps, PDGFRA mutations were identified only in $55 \%$ of the analyzed lesions. There are several explanations for such a discrepancy. Other mutational 'hot spots' or other molecular mechanisms leading to this cellular proliferation should be considered. Recent studies have shown that a detection rate of KIT and PDGFRA mutations tends to decrease with the increasing age of paraffin 


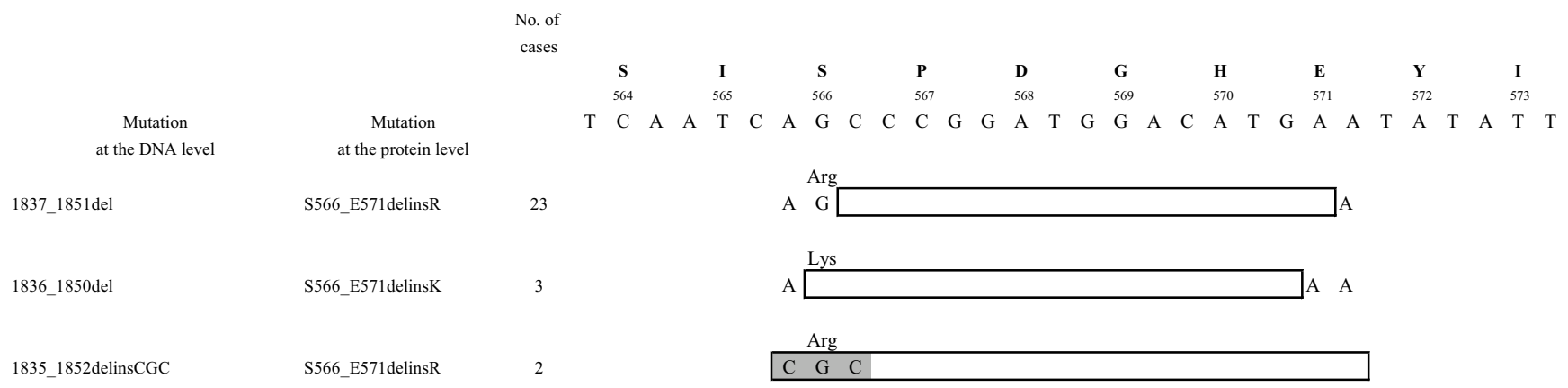

1837_1856delinsGATTGATGATC S566_I573delinsRIDDL

1

\begin{tabular}{ll|lllllllllll}
$A$ & $G$ & G & $A$ & T & T & G & A & T & G & A & T & C \\
\hline
\end{tabular}

\begin{tabular}{|c|c|c|c|c|c|c|c|c|c|c|c|c|c|c|c|c|c|c|c|c|c|c|c|c|c|c|c|c|c|c|}
\hline & & & & $\underset{556}{\mathbf{E}}$ & & & $\underset{557}{\mathbf{I}}$ & & & $\underset{558}{\mathbf{R}}$ & & & $\begin{array}{l}\mathbf{W} \\
559\end{array}$ & & & $\begin{array}{l}\mathbf{R} \\
560\end{array}$ & & & $\begin{array}{l}\mathbf{V} \\
561\end{array}$ & & $\begin{array}{c}\mathbf{I} \\
562\end{array}$ & & & $\begin{array}{l}\mathbf{E} \\
563\end{array}$ & & $\underset{564}{\mathbf{S}}$ & & & $\begin{array}{c}\mathbf{I} \\
565\end{array}$ & \\
\hline & & & G & A & A & A & $\mathrm{T}$ & $\mathrm{T}$ & $\mathrm{C}$ & G & $\mathrm{C}$ & $\mathrm{T}$ & G & G & A & G & G & $\mathrm{G}$ & $\mathrm{T} \quad \mathrm{C}$ & A & $\mathrm{T}$ & $\mathrm{T}$ & G & A A & A $\mathrm{T}$ & $\mathrm{T} \quad \mathrm{C}$ & A & A & $\mathrm{T}$ & $\mathrm{C}$ \\
\hline 1808_1828dup & I557_E563dup & 1 & G & $\mathrm{A}$ & $\mathrm{A}[$ & $\mathrm{A}$ & $\mathrm{T}$ & $\mathrm{T}$ & $\mathrm{C}$ & $\mathrm{G}$ & $\mathrm{C}$ & $\mathrm{T}$ & $\mathrm{G}$ & $\mathrm{G}$ & $\mathrm{A}$ & $\mathrm{G}$ & $\mathrm{G}$ & $\mathrm{G}$ & $\mathrm{T} \quad \mathrm{C}$ & $\mathrm{A}$ & $\mathrm{T}$ & $\mathrm{T}$ & $\mathrm{G}$ & $\mathrm{A} \quad \mathrm{A}$ & $\mathrm{A} A$ & A $T$ & $\mathrm{~T}$ & $\mathrm{C}$ & $\mathrm{G}$ & $\mathrm{C}$ \\
\hline $1821 \mathrm{~T}>\mathrm{A}$ & $561 \mathrm{~V}>\mathrm{D}$ & 1 & G & A & A & $\mathrm{A}$ & $\mathrm{T}$ & $\mathrm{T}$ & $\mathrm{C}$ & $\mathrm{G}$ & $\mathrm{C}$ & $\mathrm{T}$ & G & G & A & $\mathrm{G}$ & G & $\mathrm{G}$ & A $C$ & A & $\mathrm{T}$ & $\mathrm{T}$ & G & A A & A $\mathrm{T}$ & $\mathrm{T} \quad \mathrm{C}$ & A & A & $\mathrm{T}$ & $\mathrm{C}$ \\
\hline
\end{tabular}

Figure 2 Spectrum of PDGFRA exon 12 mutations identified in small intestinal inflammatory fibroid polyps. Empty and gray boxes indicate deletion and insertion of genetic material, respectively. Single nucleotide substitution is marked by the black box. PDGFRA wildtype sequence, codon numbers, and amino acids are shown above mutants.

blocks, most likely because of degradation of tumor DNA in archival paraffin blocks. ${ }^{22}$ Furthermore, a large normal cell component in inflammatory fibroid polyps increases the copy number of PDGFRA wild-type sequences and elevates wildtype versus mutant sequences after PCR amplification. In this study, the copy number of PDGFRA wild-type exon 12 and exon 18 sequences was reduced by cleavage of normal allele with a specific restriction endonuclease. Such a reduction of PDGFRA wild-type sequences allowed for more effective PCR amplification of the mutated allele and led to the detection of four more deletions. The detection of a single nucleotide substitution could not be improved by this strategy because there was no change in restriction sites compared with those in normal tissue. However, such mutants represented only a small fraction, 2 of $29(3.57 \%)$, when undigested tumor DNA samples were screened for PDGFRA mutations.

PDGFRA expression and oncogenic PDGFRA mutations are typically seen in gastric GISTs with epithelioid morphology. ${ }^{22}$ Although PDGFRA mutations detected in inflammatory fibroid polyps were structurally similar to those identified in gastric GISTs, inflammatory fibroid polyps should not be confused with GISTs. The pathological features of GIST and inflammatory fibroid polyps are substantially different. Small intestinal GISTs are only exceptionally detected as intraluminal polyps, and GISTs in general lack a prominent inflammatory infiltrate. ${ }^{23}$ They also express two highly specific GIST markers, KIT and DOG-1. ${ }^{20,24}$ These markers were absent in all inflammatory fibroid polyps in this study, further contrasting GISTs and fibroid polyps. Other studies have also shown similar immunohistochemical and molecular markers in different tumors. For example, KIT expression and identical activating KIT mutations were found in GISTs and in a subset of malignant melanomas. ${ }^{25}$ Thus, a diagnosis should not be taken out of the histopathological and clinicopathological context and based exclusively on immunohistochemical or molecular markers.

Earlier experimental and clinical studies have documented the oncogenic potential of PDGFRA mutations in GISTs. Table 1 summarizes these data. ${ }^{18,26,27}$ The frequency of PDGFRA exon 12 and exon 18 mutants differed substantially between small intestinal and gastric inflammatory fibroid polyps. In small intestinal tumors, almost $94 \%$ of mutations were located in PDGFRA exon 12 and represented deletion/deletion-insertions. In contrast, a majority $(62.5 \%)$ of gastric inflammatory fibroid polyps were reported to have PDGFRA exon 18 mutations, with D842V substitution being the most common; ${ }^{13}$ thus, the PDGFRA mutation pattern reported in gastric inflammatory fibroid polyps resembles the one seen in gastric GISTs. ${ }^{22}$ In contrast, PDGFRA exon 12 deletion/deletion-insertions, rarely identified in gastric inflammatory fibroid polyps and GISTs, dominated in small intestinal inflammatory fibroid polyps. The explanation and significance of this molecular difference have to be further evaluated. Two mutations, a complex deletion-insertion leading to S566_I573delinsRIDDL and I557_E563dup, were identified in this study for the first time. The latter involved the 
Table 1 Summary of type, frequency, and biological potential of PDGFRA mutations identified in gastric and small intestinal IFPs

\begin{tabular}{|c|c|c|c|c|c|c|}
\hline \multirow[b]{2}{*}{ PDGFRA mutations } & \multicolumn{2}{|c|}{$I F P$} & \multicolumn{2}{|c|}{ PDGFRA } & \multicolumn{2}{|c|}{ Studies on PDGFRA mutations in GIST } \\
\hline & Gastric $^{\mathrm{a}}$ & $\begin{array}{c}\text { Small } \\
\text { intestinal }\end{array}$ & $\begin{array}{l}\text { Activated } \\
\text { 'in vitro' }\end{array}$ & $\begin{array}{l}\text { Activated } \\
\text { 'in vivo' }\end{array}$ & $\begin{array}{l}\text { Imatinib } \\
\text { sensitivity }\end{array}$ & References \\
\hline \multicolumn{7}{|l|}{ Exon 12} \\
\hline I557_E563dup & 0 & 1 & UNK & UNK & UNK & \multirow{4}{*}{$18,26,27$} \\
\hline $561 \mathrm{~V}>\mathrm{D}$ & 1 & 1 & YES & UNK & YES & \\
\hline R560_567delinsS & 1 & 0 & UNK & UNK & UNK & \\
\hline 559_561del, 591D > H & 1 & 0 & UNK & UNK & UNK & \\
\hline S566 E571delinsR & 3 & 25 & YES & YES & YES & \multirow{3}{*}{18} \\
\hline S566_E577delinsK & 0 & 3 & UNK & UNK & UNK & \\
\hline S566_I573delinsRIDDL & 0 & 1 & UNK & UNK & UNK & \\
\hline \multicolumn{7}{|l|}{ Exon 18} \\
\hline $842 \mathrm{D}>\mathrm{V}$ & 7 & 1 & YES & YES & NO & \multirow[t]{2}{*}{$18,26,27$} \\
\hline $842 \mathrm{D}>\mathrm{I}$ & 1 & 0 & UNK & UNK & UNK & \\
\hline 842_845del & 1 & 1 & YES & YES & YES & 18 \\
\hline 845_848del & 1 & 0 & YES & UNK & YES & 18 \\
\hline
\end{tabular}

${ }^{\mathrm{a}}$ Reported earlier (Schildhaus ${ }^{13}$ ).

region earlier shown twice to be duplicated in GISTs. ${ }^{28,29}$ Although the biological potential of these two mutations has not been tested, they could also represent gain-of-function PDGFRA mutations.

Structurally similar germline PDGFRA mutations were found in the human familial GIST syndrome and in a unique patient with GIST, multiple small intestinal fibrous polyps and multiple lipomas. ${ }^{30-32}$ On the basis of illustrations, those polyps could be in the spectrum of the inflammatory fibroid polyp, although inflammatory cells were not prominent. In addition, some of the recurrent small polyps identified in patients with familial inflammatory fibroid polyps lacked inflammatory changes. ${ }^{5}$

There are three reports on the familial occurrence of inflammatory fibroid polyps. In these families, multiple, recurrent lesions developed mostly in the ileal location, with only a few gastric tumors being identified. There was a predilection to female family members. ${ }^{5,33,34}$ Subsequently, the name 'Devon polyposis syndrome' was introduced to describe such a familial condition. ${ }^{6}$ The relationship between the Devon polyposis syndrome and the mutational activation of PDGFRA is unknown. In this study, all inflammatory fibroid polyps were solitary lesions and a majority of them were located in the ileum. No data suggesting familial occurrence were available. In contrast to the Devon polyposis syndrome, small intestinal inflammatory fibroid polyps were diagnosed equally in male and female patients.

The tyrosine kinase inhibitor, imatinib mesylate, also known as STI 571 or Gleevec/Glivec (Novartis) has been used successfully to inhibit the proliferation of clinically advanced and metastatic GISTs. ${ }^{35,36}$ GIST PDGFRA exon 12 mutants respond to this tyrosine kinase inhibitor treatment. However, tumors with PDGFRA D842V substitution, which corresponds to imatinib-resistant KIT D816V mutation reported in human mastocytosis, are resistant to imatinib. ${ }^{18,27}$ Although most cases of small intestinal inflammatory fibroid polyps require surgery to relieve the intestinal obstruction, tyrosine kinase inhibitor treatment might be a consideration in selected cases, such as poor surgical-risk patients.

Several theories on histogenesis of inflammatory fibroid polyps have been coined on the basis of ultrastructural and immunohistochemical studies. However, the consistent PDGFRA expression seen in gastric and small intestinal inflammatory fibroid polyps points to the fact that these tumors might develop from a subset of PDGFRA-positive mesenchymal cells.

Clusters of PDGFRA-positive mesenchymal cells distributed along the villus basement membrane, referred to as 'villus clusters', have been identified in mouse embryos. These cells are crucial for villus formation. Moreover, it is known that some PDGFRA-positive cells remain at the same locations in adult intestine; however, it is not clear whether they represent the quiescent mesenchymal stem cells or progenitor cells, or simply exert a differentiated function. ${ }^{37}$

PDGFRA-positive mesenchymal cells distributed along the villus basement membrane are PDGFA/ PDGFRA-dependent, and lack of PDGFA or PDGFRA resulted in a progressive depletion of these cells in PDGFA- and PDGFRA-deficient mice. ${ }^{37}$ Thus, it seems plausible that PDGFRA-activating mutations can lead to an uncontrolled proliferation of such cells. A hypothesis that PDGFRA-positive mesenchymal cells could be the possible ancestral cells for inflammatory fibroid polyps could mirror the model of GIST pathogenesis, which assumes that GISTs are derived from KIT-dependent Cajal cells driven into uncontrolled proliferation by gain-offunction KIT mutations. ${ }^{38}$ Nevertheless, to prove such a hypothesis, a mouse model with mutationally activated PDGFRA should be developed. 
In summary, we have shown that small intestinal inflammatory fibroid polyps, similar to gastric inflammatory fibroid polyps, express PDGFRA and often have oncogenic PDGFRA mutations. Thus, the inflammatory fibroid polyp should be considered as a PDGFRA-driven benign neoplasm. We have also postulated that inflammatory fibroid polyps may develop from PDGFRA-positive mesenchymal cells distributed along the villus basement membrane. The term inflammatory fibroid polyp was introduced by Helwig and Ranier ${ }^{2}$ to indicate the possible non-neoplastic nature of this lesion; however, in the scope of current research, the term inflammatory fibroid tumor indicating the neoplastic nature of this lesion may be more appropriate.

\section{References}

1 Vanek J. Gastric submucosal granuloma with eosinophilic infiltration. Am J Pathol 1949;25:397-411.

2 Johnstone JM, Morson BC. Inflammatory fibroid polyp of the gastrointestinal tract. Histopathology 1978;2: 349-361.

3 Helwig EB, Ranier A. Inflammatory fibroid polyps of the stomach. Surg Gynecol Obstet 1953;96:335-367.

4 Shimer GR, Helwig EB. Inflammatory fibroid polyps of the intestine. Am J Clin Pathol 1984;81:708-714.

5 Spencer D. Recurrent familial inflammatory fibroid polyps of the small intestine. J Clin Pathol 1969;22:743.

6 Allibone RO, Nanson JK, Anthony PP. Multiple and recurrent inflammatory fibroid polyps in a Devon family ('Devon polyposis syndrome'): an update. Gut 1992;33:1004-1005.

7 Kolodziejczyk P, Yao T, Tsuneyoshi M. Inflammatory fibroid polyp of the stomach. A special reference to an immunohistochemical profile of 42 cases. Am J Surg Pathol 1993;17:1159-1168.

8 Hasegawa T, Yang P, Kagawa N, et al. CD34 expression by inflammatory fibroid polyps of the stomach. Mod Pathol 1997;10:451-456.

9 Wille P, Borchard F. Fibroid polyps of intestinal tract are inflammatory-reactive proliferations of CD34-positive perivascular cells. Histopathology 1998;32: 498-502.

10 Kim MK, Higgins J, Cho EY, et al. Expression of CD34, bcl-2, and kit in inflammatory fibroid polyps of the gastrointestinal tract. Appl Immunohistochem Mol Morphol 2000;8:147-153.

11 Pantanowitz L, Antonioli DA, Pinkus GS, et al. Inflammatory fibroid polyps of the gastrointestinal tract: evidence for a dendritic cell origin. Am J Surg Pathol 2004;28:107-114.

12 Santos Gda C, Alves VA, Wakamatsu A, et al. Inflammatory fibroid polyp: an immunohistochemical study. Arq Gastroenterol 2004;41:104-107.

13 Schildhaus HU, Cavlar T, Binot E, et al. Inflammatory fibroid polyps harbour mutations in the plateletderived growth factor receptor alpha (PDGFRA) gene. J Pathol 2008;216:176-182.

14 Roberts WM, Look AT, Ruossel MF, et al. Tandem linkage of human CSF-1 receptor (c-fms) and PDGF receptor genes. Cell 1989;55:655-661.
15 Stenman G, Eriksson A, Claesson-Welsh L. Human PDGFA receptor gene maps to the same region on chromosome 4 as the KIT oncogene. Genes Chromosomes Cancer 1989;1:155-158.

16 Pawson T. Regulation and targets of receptor tyrosine kinases. Eur J Cancer 2002;38:S3-S10.

17 Fletcher JA. Role of KIT and platelet-derived growth factor receptors as oncoproteins. Semin Oncol 2004;31(Suppl 6):4-11.

18 Heinrich MC, Corless CL, Duensing A, et al. PDGFRA activating mutations in gastrointestinal stromal tumors. Science 2003;299:708-710.

19 Lasota J, Dansonka-Mieszkowska A, Sobin LH, et al. A great majority of GISTs withPDGFRA mutations represents gastric tumors of low or no malignant potential. Lab Invest 2004;84:874-883.

20 Miettinen M, Sobin LH, Sarlomo-Rikala M. Immunohistochemical spectrum of GISTs at different sites and their differential diagnosis with a reference to CD117 (KIT). Mod Pathol 2000;13:1134-1142.

21 Lasota J, Stachura J, Miettinen M. GISTs with PDGFRA exon 14 mutations represent subset of clinically favorable gastric tumors with epithelioid morphology. Lab Invest 2006;86:94-100.

22 Lasota J, Miettinen M. Clinical significance of oncogenic KIT and PDGFRA mutations in gastrointestinal stromal tumours. Histopathology 2008;53:245-266.

23 Miettinen M, Makhlouf HR, Sobin LH, et al. Gastrointestinal stromal tumors (GISTs) of the jejunum and ileum - a clinicopathologic, immunohistochemical and molecular genetic study of 906 cases prior to imatinib with long-term follow-up. Am J Surg Pathol 2006;30:625-641.

24 Espinosa I, Lee CH, Kim MK, et al. A novel monoclonal antibody against DOG1 is a sensitive and specific marker for gastrointestinal stromal tumors. Am J Surg Pathol 2008;32:210-218.

25 Curtin JA, Busam K, Pinkel D, et al. Somatic activation of KIT in distinct subtypes of melanoma. J Clin Oncol 2006;24:4340-4346.

26 Hirota S, Ohashi A, Nishida T, et al. Gain-of-function mutations of platelet-derived growth factor receptor alpha gene in gastrointestinal stromal tumors. Gastroenterology 2003;125:660-667.

27 Debiec-Rychter M, Sciot R, Le Cesne A, et al. KIT mutations and dose selection for imatinib in patients with advanced gastrointestinal stromal tumors. Eur J Cancer 2006;42:1093-1103.

28 Daum O, Grossmann P, Vanecek T, et al. Diagnostic morphological features of PDGFRA-mutated gastrointestinal stromal tumors: molecular genetic and histologic analysis of 60 cases of gastric gastrointestinal stromal tumors. Ann Diagn Pathol 2007;11:27-33.

29 Haller F, Happel N, Schulten H-J, et al. Site-dependent differential KIT and PDGFRA expression in gastric and intestinal stromal tumors. Mod Pathol 2007;20: 1103-1111.

30 Chompret A, Kannengiesser C, Barrois $\mathrm{M}$, et al. PDGFRA germline mutation in a family with multiple cases of gastrointestinal stromal tumor. Gastroenterology 2004;126:318-321.

31 de Raedt T, Cools J, Debiec-Rychter M, et al. Intestinal neurofibromatosis is a subtype of familial GIST and results from dominant activating mutation in PDGFRA. Gastroenterology 2006;131:1907-1912.

32 Pasini B, Matyakhina L, Bei $\mathrm{T}$, et al. Multiple gastrointestinal stromal tumors caused by platelet- 
derived growth factor receptor á gene mutations: a case associated with a germline V561D defect. J Clin Endocrinol Metab 2007;92:3728-3732.

33 Anthony PP, Morris DS, Vowles KD. Multiple and recurrent inflammatory fibroid polyps in three generations of a Devon family: a new syndrome. Gut 1984;25: 854-862.

34 Bayle S, Rossi P, Bagneres D, et al. Ileum inflammatory fibroid polyp revealed by intussusception. About one familial case. Rev Med Interne 2005;26:233-237.

35 Joensuu H, Roberts PJ, Sarlomo-Rikala M, et al. Effect of tyrosine kinase inhibitor STI571 in a patient with a metastatic gastrointestinal stromal tumor. N Engl J Med 2001;344:1052-1056.
36 Demetri GD. Identification and treatment of chemoresistant inoperable or metastatic GIST: experience with the selective tyrosine kinase inhibitor imatinib mesylate (STI571). Eur J Cancer 2002;38(Suppl 5): S52-S59.

37 Karlsson L, Lindahl P, Heath JK, et al. Abnormal gastrointestinal development in PDGF-A and PDGFR(alpha) deficient mice implicates a novel mesenchymal structure with putative instructive properties in villus morphogenesis. Development 2000;127: 3457-3466.

38 Hirota S, Isozaki K, Moriyama Y, et al. Gain-of-function mutations of c-kit in human gastrointestinal stromal tumors. Science 1998;279:577-580. 